\title{
MORTALITY RESPONSES OF THE BROWNHEADED AND BLACK-LYRE LEAFROLLERS TO CONTROLLED ATMOSPHERE AND AIR COLD STORAGE TREATMENTS FOR KIWIFRUIT
}

\author{
D.C. WHITING, L.E. JAMIESON and P.G. CONNOLLY \\ The Horticulture and Food Research Institute of New Zealand Ltd, \\ Private Bag 92169, Auckland, New Zealand
}

\begin{abstract}
The mortality responses of the brownheaded leafroller (Ctenopseustis obliquana) and the black-lyre leafroller (Cnephasia jactatana) to controlled atmosphere cold storage (CACS) and air cold storage (CS) treatments for kiwifruit were investigated. CACS $\left(2 \% \mathrm{O}_{2}\right.$, $5 \% \mathrm{CO}_{2}$ at $0^{\circ} \mathrm{C}$ ) killed both leafrollers faster than $\mathrm{CS}\left(\right.$ at $0^{\circ} \mathrm{C}$ ). The brownheaded leafroller was more tolerant of both storage treatments than the black-lyre leafroller. Third and 5 th instars were generally more tolerant of storage treatments than 1st instars, which in turn outlived eggs. This work predicts both brownheaded and black-lyre leafrollers should be completely controlled by 4 weeks CACS or 9 weeks CS.

Keywords: Ctenopseustis obliquana, Cnephasia jactatana, controlled atmosphere, cold storage, kiwifruit.
\end{abstract}

\section{INTRODUCTION}

New Zealand kiwifruit (Actinidia deliciosa cv. Hayward) are currently exported to over 60 countries, each with different quarantine regulations. Exports are either denied entry or fumigated with chemicals such as methyl bromide when live quarantine pests are intercepted by quarantine agencies. With the industry-wide implementation of KiwiGreen and the consequent reduction in use of chemical pesticides pre-harvest, the diversity of quarantine pests being found on kiwifruit after harvest is increasing. This in turn is beginning to have impact on market access. Such market access problems could be minimised by developing postharvest treatments, ideally acceptable under both KiwiGreen and organic production systems, which control quarantine pests without detrimentally affecting the fruit.

Controlled atmosphere cold storage (CACS) and air cold storage (CS) are used by the kiwifruit industry to extend the postharvest storage life of the fruit. Kiwifruit are held on average for 18-20 weeks under $\operatorname{CACS}\left(2 \% \mathrm{O}_{2}, 5 \% \mathrm{CO}_{2}, 0^{\circ} \mathrm{C}\right)$, or 14-18 weeks under CS (air, $0^{\circ} \mathrm{C}$ ). While the effects of these storage conditions on fruit quality (Harman and McDonald 1989), and the incidence of fungal diseases (Manning and Lallu 1997) have been assessed, the impact of these regimes on the survival of quarantine pests possibly present on the fruit has not been studied. Long exposures of similar storage conditions are known to control a number of leafrollers of quarantine importance on New Zealand apples (Waddell et al. 1990) and stonefruit (Whiting and Hoy 1997).

We determined the time-mortality responses of the brownheaded leafroller (Ctenopseustis obliquana) and the black-lyre leafroller (Cnephasia jactatana) to the commercial CACS and CS conditions used for Hayward kiwifruit.

\section{Insects and fruit}

\section{METHODS}

Unsprayed kiwifruit (count size 30-33) were supplied from the Te Puke Research Orchard and stored at $0^{\circ} \mathrm{C}$ until required. Two days before inoculation with insects, all fruit were transferred to $20^{\circ} \mathrm{C}$. To aid subsequent oviposition and larval establishment, 
the epidermal hairs were removed from the fruit with a soft brush and each fruit was pricked 10 times with a pin to facilitate larval penetration, the day before inoculation.

Brownheaded leafroller and black-lyre leafroller were obtained from laboratory cultures maintained at HortResearch, Auckland, at $20 \pm 1{ }^{\circ} \mathrm{C}, 60 \% \mathrm{RH}$ and a photoperiod of $16: 8 \mathrm{~h}$ (light:dark). The two leafroller species were treated on separate occasions. Sixteen fruit (one sample time of one replicate of one life stage for one leafroller species) were placed on a modified fibre tray inside a fine fabric mesh bag that prevented insect escape. Kiwifruit were infested with either 3-day old eggs, 1st, 3 rd or 5th instar larvae using techniques previously described by Hoy and Whiting (1998). Each bag contained one life stage (>100 eggs or $>60$ larvae). Each bag was sealed and placed inside a 20-litre plastic treatment bucket. Two life stages of the same leafroller species were exposed to treatment in each bucket. A metal mesh tripod that prevented insect and fruit compression separated the bags in each bucket. Infested kiwifruit were kept at $20 \pm 1{ }^{\circ} \mathrm{C}, 70 \% \mathrm{RH}, 16: 8 \mathrm{~h}$ light:dark for a further 3 days to facilitate larval establishment in preferred locations before storage treatment.

\section{Storage procedures}

Experiments were carried out in 45 treatment buckets located inside a cold store with an internal volume of $9.71 \mathrm{~m}^{3}(2.1 \times 2.15 \times 2.15 \mathrm{~m}, \mathrm{~L} \times \mathrm{W} \times \mathrm{H})$ and refrigeration unit rated at 545 nominal watts. Bucket lids were modified with a gas inlet and outlet port and a rubber septum to enable gas sampling. Twenty seven buckets ( 3 replicates of 9 buckets, CACS) were connected to a controlled atmosphere generated from an Isolcell nitrogen separator (Isolcell Italia, Laives, Italy) and bottles of food grade $\mathrm{CO}_{2}$ (BOC Gases New Zealand Limited). The remaining 18 buckets (3 replicates of 6 buckets, CS) were connected to a low-pressure compressed air supply. Gas supply was mixed in a manifold and bubbled through a humidifier before being split to all treatment buckets of a replicate of the appropriate treatment type. The flow rate through each bucket $(333 \mathrm{ml} / \mathrm{min}$ ) ensured one complete atmosphere change every hour. Bucket outlets of each replicate of the appropriate treatment type were similarly manifolded together into a common outlet to ensure even back pressure.

All treatment buckets were attached simultaneously to gas inlet lines inside the cold store (exposure time $=0 \mathrm{~d}$ ) and atmosphere establishment and fruit cooling commenced immediately. Cold store air temperature was measured every hour with a thermistor probe located outside the treatment buckets at fruit level and connected to a Squirrel data logger (Grant 1206 model). It took $7 \mathrm{~h}$ to reduce the temperature to $<0.5^{\circ} \mathrm{C}$ and this was maintained at $0.15 \pm 0.01^{\circ} \mathrm{C}$ during the trial. Fruit surface and centre temperatures were measured in the 6 buckets allotted to the longest exposure of each storage treatment ( 3 buckets each). From the fruit starting temperature of $20 \pm 1^{\circ} \mathrm{C}$, the kiwifruit surface, measured with a thermistor probe held onto the surface of one fruit in each bucket with teflon tape (Ceelon), reached target temperature after $41 \mathrm{~h}$. After equilibrium, mean fruit surface temperature within the buckets was 0.26 $\pm 0.003^{\circ} \mathrm{C}$. The fruit centre, measured with a thermistor probe inserted into the centre of one kiwifruit in each bucket, took on average $45 \mathrm{~h}$ to attain target temperature and after this period averaged $0.30 \pm 0.003^{\circ} \mathrm{C}$. Relative humidity, measured with a capacity humidity probe ( $\mathrm{VH}-\mathrm{H}$, Lee-Integer, England) in two buckets, was $>90 \%$ at equilibrium. The atmosphere composition in all buckets was sampled and analysed throughout each experiment using a paramagnetic $\mathrm{O}_{2}$ sensor (Servomex 1111D) and an infrared $\mathrm{CO}_{2}$ sensor (Servomex 1505). The composition of $\mathrm{O}_{2}$ and $\mathrm{CO}_{2}$ in the buckets was calculated by calibration of the sensors with a standard reference gas containing either $1.67 \% \mathrm{O}_{2}$ and $5 \% \mathrm{CO}_{2}$ (vol:vol) (CACS treatment), or air $(20.95 \%$ $\mathrm{O}_{2}$ and $0.03 \% \mathrm{CO}_{2}$ (vol:vol) (CS treatment). Target $\mathrm{O}_{2}$ and $\mathrm{CO}_{2}$ concentrations for CACS treatment (actual mean composition $2.06 \pm 0.01 \% \mathrm{O}_{2}, 5.04 \pm 0.02 \% \mathrm{CO}_{2}$ ) were established approximately 6 and $3 \mathrm{~h}$ into the treatment, respectively. All experiments were performed in darkness.

Samples were removed at exposure times designed to cover $20-100 \%$ mortality. After treatment, all insect-infested fruit were held at $20 \pm 1^{\circ} \mathrm{C}, 70 \% \mathrm{RH}, 16: 8 \mathrm{~h}$ (light:dark) until mortality assessment 10 days (eggs) and 2 to 3 days later (larvae). Three buckets of control fruit were exposed to air at $20 \pm 1^{\circ} \mathrm{C}, 70 \% \mathrm{RH}, 16: 8 \mathrm{~h}$ 
(light:dark) for 3 days. Kiwifruit were dissected and individual larvae were examined under a binocular microscope at 30× magnification. Egg mortality was calculated by counting the number of hatched and unhatched eggs. Larvae were probed with a pin and recorded as live (movement) or dead (no movement).

Statistical analyses

Time-mortality data for each replicate were fitted using the complementary log$\log$ (clog-log) model (Preisler and Robertson 1989) with time as the explanatory variable to derive estimated days for 50, 99 and 99.9968 (probit 9) \% mortality $\left(\mathrm{LT}_{50}\right.$, $\mathrm{LT}_{99}$ and $\mathrm{LT}_{99.9968}$, respectively). These estimates were calculated as the time to achieve a mortality of $c+(1-c) \times m$, where $c$ was the control mortality and $m$ the estimated proportion mortality. For each life stage of each leafroller species a geometric mean LT and its associated standard error (SEM) were estimated, from which a $95 \%$ confidence interval (CI) was calculated. Non-overlap of the $95 \%$ CIs is equivalent to a test for difference ca $\mathrm{P}=0.01$.

\section{Controlled atmosphere cold storage}

\section{RESULTS}

Percentage mortality increased steadily with increasing exposure time to CACS for both species (Fig. 1). The brownheaded leafroller was more tolerant of CACS than was the black-lyre leafroller.

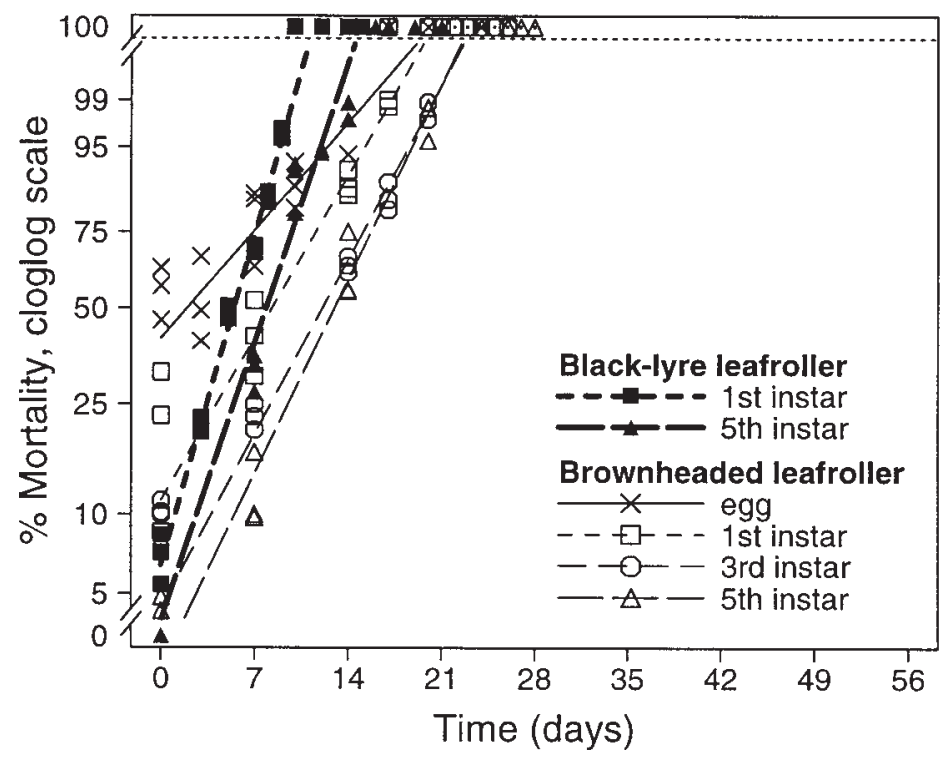

\section{FIGURE 1: Mean percentage mortality of brownheaded and black-lyre leafrollers exposed to CACS on kiwifruit.}

Tolerance of CACS treatment tended to increase with increase in insect age. The percentage mortality of brownheaded leafroller eggs was initially higher than 3rd and 5 th instars, with 1 st instars displaying an intermediary response. However, as the CACS treatment progressed, the mortality responses of the life stages statistically converged (Table 1). This convergence was not evident in the black-lyre leafroller. To attain a probit 9 level of security, 5th instar larvae of the brownheaded and black-lyre leafrollers required CACS exposures of $23.5 \pm 0.8$ and $15.3 \pm 0.4$ days, respectively. 
TABLE 1: Mean $\mathrm{LT}_{50}, \mathrm{LT}_{99}$ and $\mathrm{LT}_{\mathbf{9 9 . 9 9 6 8}}$ (days) derived from time-mortality data of brownheaded and black-lyre leafrollers exposed to CACS on kiwifruit.

\begin{tabular}{llcccc}
\hline Leafroller & $\begin{array}{l}\text { Life } \\
\text { stage }\end{array}$ & $\mathrm{n}_{\text {total }}$ & $\begin{array}{c}\text { Mean LT } \\
(95 \% \mathrm{CI})\end{array}$ & $\begin{array}{c}\text { Mean LT } \\
(95 \% \mathrm{CI})\end{array}$ & $\begin{array}{c}\text { Mean LT } \\
(95 \% \mathrm{CI})\end{array}$ \\
\hline Brownheaded & egg & 3741 & $7.4(6.3-8.7)$ & $15.8(14.5-17.2)$ & $20.5(18.5-22.8)$ \\
& 1st & 2949 & $9.5(8.1-11.3)$ & $17.4(16.0-19.0)$ & $21.1(19.0-23.4)$ \\
& 3rd & 2975 & $12.7(10.8-15.0)$ & $20.5(18.9-22.3)$ & $24.1(21.7-26.7)$ \\
& 5th & 1741 & $13.3(11.3-15.7)$ & $20.4(18.7-22.1)$ & $23.5(21.2-26.1)$ \\
Black-lyre & 1st & 2407 & $5.7(5.4-6.0)$ & $9.9(9.5-10.3)$ & $11.8(11.2-12.5)$ \\
& 5th & 3085 & $7.9(7.5-8.3)$ & $13.0(12.5-13.6)$ & $15.3(14.4-16.1)$ \\
\hline
\end{tabular}

\section{Cold storage}

CS took longer than CACS to control both leafrollers (Figs 1 and 2). As found with CACS, the brownheaded leafroller was more tolerant of CS than was the black-lyre leafroller, and older larvae survived longer than 1st instars, which in turn outlived eggs (Fig. 2). However, the life stages displayed a wider range of mortality responses when exposed to this storage treatment than to CACS.

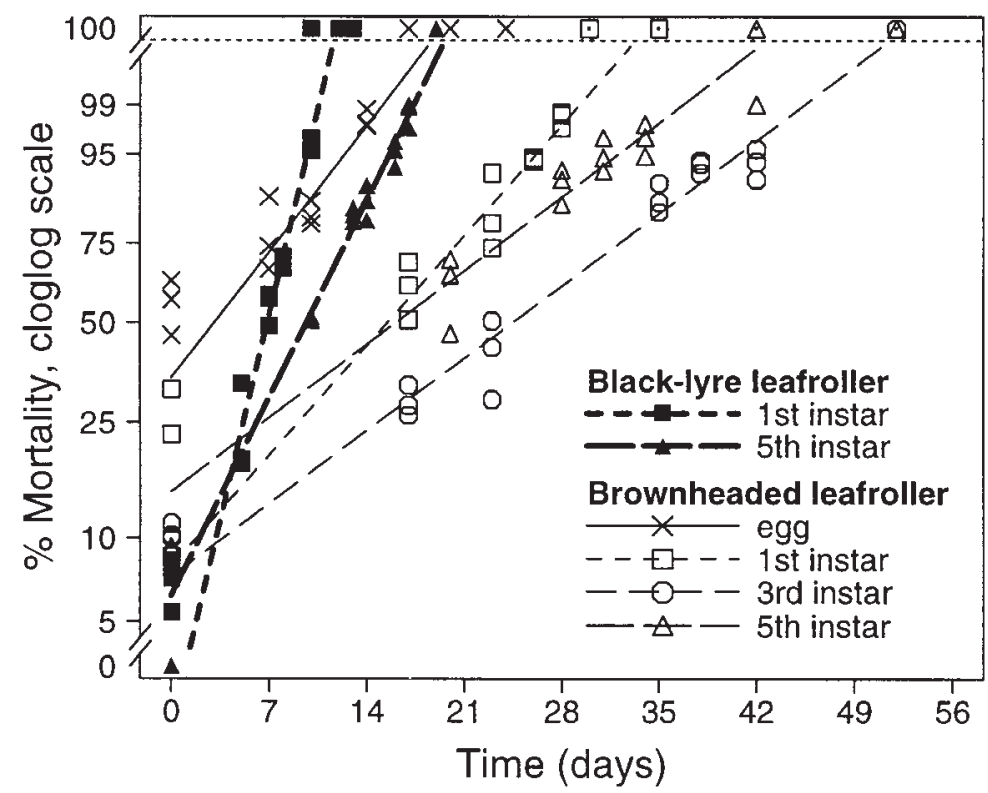

FIGURE 2: Mean percentage mortality of brownheaded and black-lyre leafrollers exposed to CS on kiwifruit.

Unexpectedly, brownheaded leafroller 3rd instars were more tolerant of CS treatment than 5 th instars. However, the CS exposure time required by 3 rd instars for probit 9 level mortality $(54.5 \pm 1.1 \mathrm{~d})$, while still higher, was not statistically different from that required by the more mature larvae (Table 2). Black-lyre leafroller 5 th instar larvae required a $20.6 \pm 0.3$ days CS exposure to attain this high level of mortality. 

TABLE 2: $\quad$ Mean $\mathrm{LT}_{50}, \mathrm{LT}_{99}$ and $\mathrm{LT}_{\mathbf{9 9 . 9 9 6 8}}$ (days) derived from time-mortality data of brownheaded and black-lyre leafrollers exposed to CS on kiwifruit.

\begin{tabular}{llcccc}
\hline Leafroller & $\begin{array}{l}\text { Life } \\
\text { stage }\end{array}$ & $\mathrm{n}_{\text {total }}$ & $\begin{array}{c}\text { Mean LT } \\
(95 \% \mathrm{CI})\end{array}$ & $\begin{array}{c}\text { Mean LT } \\
(95 \% \mathrm{CI})\end{array}$ & $\begin{array}{c}\text { Mean LT } \\
(95 \% \mathrm{CI})\end{array}$ \\
\hline Brownheaded & egg & 3182 & $7.9(6.7-9.3)$ & $16.4(15.1-17.8)$ & $21.2(19.1-23.5)$ \\
& 1st & 1626 & $17.3(14.7-20.5)$ & $29.7(27.3-32.2)$ & $35.4(31.9-39.3)$ \\
& 3rd & 1654 & $26.2(22.2-30.9)$ & $45.7(42.0-49.7)$ & $54.5(49.1-60.4)$ \\
Black-lyre & 5th & 2049 & $16.3(13.8-19.3)$ & $36.9(34.0-40.1)$ & $45.9(41.3-50.9)$ \\
& 1st & 1664 & $7.1(6.7-7.4)$ & $10.5(10.1-11.0)$ & $12.1(11.4-12.7)$ \\
& 5th & 2190 & $9.8(9.3-10.4)$ & $17.4(16.7-18.1)$ & $20.6(19.5-21.8)$ \\
\hline
\end{tabular}

\section{DISCUSSION}

This study is the first to determine the mortality responses of the black-lyre leafroller to CACS and CS. However, the response of brownheaded leafroller larvae to these types of storage treatments of apples has been previously examined on two studies using commercial facilities. All $\mathrm{LT}_{99}$ estimates calculated from CACS trials on the same three larval stages (Waddell et al. 1990) exceeded those values estimated in this study. However, this earlier work used a more moderate CACS regime $(3.2 \%$ $\mathrm{O}_{2}, 2.9 \% \mathrm{CO}_{2}, 0.6^{\circ} \mathrm{C}$ ) and LT estimates were based, at best, on data from only three relevant exposure times. Batchelor et al. (1985) found 3rd instar brownheaded leafroller could survive 17 weeks CS at the higher temperature of $0.9^{\circ} \mathrm{C}$.

Our work determined that both the brownheaded and black-lyre leafrollers on harvested kiwifruit could be completely controlled by 4 weeks CACS or 9 weeks CS. Although our experimental facility attained the target treatment conditions much faster than a commercial store, these exposure times are well within the average times export kiwifruit is standardly held in these regimes. Even if the fruit is stored for shorter periods of time, significant leafroller mortality and probably complete control of more susceptible species (such as the black-lyre leafroller) or immature life stages (such as eggs and 1st instars) may result.

Future research should (a) determine the mortality responses of other quarantine pests intercepted on export kiwifruit to these storage treatments, (b) demonstrate the mortality responses of relevant insects on a larger scale in commercial CACS and CS facilities and (c) assess the tolerance of new kiwifruit cultivars, e.g. A. chinensis and A. arguta, to these storage treatments.

\section{ACKNOWLEDGEMENTS}

We thank A.M. Barrington for supplying leafrollers, and S.E. Lewthwaite, X. Meier, P.A. Boxen, V.A. Bush, R.J. Petry, G.K. Clare, D.J. Rogers, P.R. Dentener, and Z.M. Whiting for technical assistance. This work was funded by the Foundation for Research, Science and Technology contract CO6822 and Kiwifruit New Zealand Research Limited.

\section{REFERENCES}

Batchelor, T.A, O’Donnell, R.L. and Roby, J.J., 1985. The efficacy of controlled atmosphere coolstorage in controlling leafroller species. Proc. 38th N.Z. Weed and Pest Control Conf.: 53-55.

Harman, J.E. and McDonald, B., 1989. Controlled atmosphere storage of kiwifruit. Effect on fruit quality and composition. Scientia Hort. 37: 303-315.

Hoy, L.E. and Whiting, D.C., 1998. Mortality responses of three leafroller(Lepidoptera: Tortricidae) species on kiwifruit to a high-temperature controlled atmosphere treatment. N.Z. J. Crop Hort. Sci. 26: 11-15.

Manning, M.A. and Lallu, N., 1997. Fungal diseases of kiwifruit stored in controlled atmosphere conditions in New Zealand. Acta Hort. 444: 725-731. 
Preisler, H.K. and Robertson, J.L., 1989. Analysis of time-dose-mortality data. J. Econ. Ent. 82: 1534-1542.

Waddell, B.C., Dentener, P.R. and Batchelor, T.A., 1990. Time-mortality response of leafrollers exposed to commercial controlled atmosphere coolstorage.Proc. 43rd N.Z. Weed and Pest Control Conf:: 328-333.

Whiting, D.C. and Hoy, L.E., 1997. Mortality response of lightbrown apple moth to a controlled atmosphere cold storage treatment for apricots. Proc. 50th N.Z. Plant Prot. Conf.: 431-435. 